\title{
High-order Techniques for Calculating Surface Tension Forces
}

\author{
M. Sussman and M. Ohta
}

\begin{abstract}
In this paper we develop further the "height fraction" technique for computing curvature directly from volume fractions. In particular we, (1) develop a systematic approach for calculating curvature from volume fractions which is accurate to any order, and (2) we test the second-order "height fraction" technique on the following two-phase problems: (1) the break-up of a cylindrical column of liquid due to Rayleigh-capillary instability, (2) surface tension induced droplet oscillations and (3) the steady motion of gas bubbles rising in liquid.
\end{abstract}

Keywords. Curvature, volume-of-fluid method, level set, multiphase flow.

\section{Introduction}

Numerical algorithms for calculating surface tension have been presented from the perspective of front tracking algorithms [14, 26, 25, 28, 16], Volume-of-Fluid algorithms $[3,7,1,18,8]$, level set methods $[24,12,13]$, and various hybrid methods $[23,20,6]$. The ability to accurately calculate surface tension can be important for modeling the impact of drops on surfaces, contact line dynamics, bubble motion, and the break-up of liquid jets. In our previous work [20], a second-order coupled level set and volume of fluid method was presented for calculating bubble growth and collapse. In that work, the "height fraction" technique[9] was employed to accurately calculate curvature directly from volume fractions. In this paper, we present additional calculations further validating the second-order method originally proposed in [20]. Furthermore, we demonstrate that the "height fraction" technique can be extended to calculate curvature to any order of accuracy.

Conventional wisdom would have it that only a level set representation of an interface is capable of having a very high-order accurate method for extracting

Work supported in part by NSF grant number 0242524 (U.S. Japan Cooperative Science).

Work supported in part by JSPS. 
the interface curvature. For example, a "spectral" level set approach was presented by[21]. Previous methods for extracting curvature from volume fractions have been proposed by Chorin [4] (osculating circle technique), Poo and Ashgriz [15], Aleinov and Puckett [1] (convolution technique), Williams et al. [27], Renardy et al. [17] ("PROST"), and the "height fraction" approach $[9,20,7,5]$. The challenge in accurately calculating curvature from the volume-of-fluid function $F$ is the fact that $F$ transitions sharply from 0 (computational cell containing only gas) to 1 (computational cell containing only liquid). Of all the approaches for calculating curvature from volume fractions, the "height fraction" approach is the most direct, and, unlike convolution techniques, it is "localized."

In this paper we present a systematic approach to extending the "height fraction" approach to any order of accuracy (i.e., higher than second order).

\section{Curvature discretization using "height fractions"}

The curvature of an interface is computed to second- or fourth-order accuracy directly from the volume fractions. The method is based on reconstructing the "height" function directly from the volume fractions [9]. Without loss of generality, we assume that the free surface is oriented more horizontal than vertical. For a second-order curvature algorithm, a $3 \times 3 \times 7$ stencil of volume fractions is constructed about cell $(i, j, k)$. For the fourth-order algorithm, a $5 \times 5 \times 13$ stencil of volume fractions is constructed about cell $(i, j, k)$. The $3 \times 3(5 \times 5$ for fourthorder) vertical sums, $F_{i^{\prime}, j^{\prime}}, i^{\prime}=-1 \ldots 1, j^{\prime}=-1 \ldots 1$, are exact integrals of the height function $h(x, y)$ (up to a constant); i.e.,

$$
F_{i^{\prime}, j^{\prime}} \Delta x \Delta y \Delta z=\int_{x_{i+i^{\prime}-1 / 2}}^{x_{i+i^{\prime}+1 / 2}} \int_{y_{j+j^{\prime}-1 / 2}}^{y_{j+j^{\prime}+1 / 2}} h(x, y) d x d y+C .
$$

It can be shown that $\Delta z\left(F_{1,0}-F_{-1,0}\right) /(2 \Delta x)$ is a second-order approximation to $h_{x}\left(x_{i}, y_{j}\right)$ and that $\Delta z\left(F_{1,0}-2 F_{0,0}+F_{-1,0}\right) / \Delta x^{2}$ is a second-order approximation to $h_{x x}\left(x_{i}, y_{j}\right)$. In general, one expands $h(x, y)$ in a Taylor series,

$$
\begin{aligned}
& h(x, y)=h\left(x_{i}, y_{j}\right)+\left(x-x_{i}\right) h_{x}\left(x_{i}, y_{j}\right)+\frac{1}{2}\left(x-x_{i}\right)^{2} h_{x x}\left(x_{i}, y_{j}\right) \\
& \quad+\frac{1}{6}\left(x-x_{i}\right)^{3} h_{x x x}\left(x_{i}, y_{j}\right)+\frac{1}{24}\left(x-x_{i}\right)^{4} h_{x x x x}\left(x_{i}, y_{j}\right)+\left(y-y_{j}\right) h_{y}\left(x_{i}, y_{j}\right) \\
& \quad+\frac{1}{2}\left(y-y_{j}\right)^{2} h_{y y}\left(x_{i}, y_{j}\right)+\frac{1}{6}\left(y-y_{j}\right)^{3} h_{y y y}\left(x_{i}, y_{j}\right)+\frac{1}{24}\left(y-y_{j}\right)^{4} h_{y y y y}\left(x_{i}, y_{j}\right) \\
& \quad+\left(x-x_{i}\right)\left(y-y_{j}\right) h_{x y}\left(x_{i}, y_{j}\right)+\frac{1}{2}\left(x-x_{i}\right)^{2}\left(y-y_{j}\right) h_{x x y}\left(x_{i}, y_{j}\right) \\
& \quad+\frac{1}{2}\left(x-x_{i}\right)\left(y-y_{j}\right)^{2} h_{x y y}\left(x_{i}, y_{j}\right)+\frac{1}{4}\left(x-x_{i}\right)^{2}\left(y-y_{j}\right)^{2} h_{x x y y}\left(x_{i}, y_{j}\right) \\
& \quad+\text { higher-order terms. }
\end{aligned}
$$


After integrating the Taylor series expansion of $h(x, y)$ and using $(2.1)$, one has

$$
\begin{aligned}
& F_{i^{\prime}, j^{\prime}} \Delta z=h\left(x_{i+i^{\prime}}, y_{j+j^{\prime}}\right)+\frac{1}{24} \Delta x^{2} h_{x x}\left(x_{i+i^{\prime}}, y_{j+j^{\prime}}\right) \\
& \quad+\frac{1}{(16)(120)} \Delta x^{4} h_{x x x x}\left(x_{i+i^{\prime}}, y_{j+j^{\prime}}\right)+\frac{1}{24} \Delta y^{2} h_{y y}\left(x_{i+i^{\prime}}, y_{j+j^{\prime}}\right) \\
& \quad+\frac{1}{(16)(120)} \Delta y^{4} h_{y y y y}\left(x_{i+i^{\prime}}, y_{j+j^{\prime}}\right)+\frac{1}{(24)(24)} \Delta x^{2} \Delta y^{2} h_{x x y y}\left(x_{i+i^{\prime}}, y_{j+j^{\prime}}\right) \\
& \quad+\text { higher-order terms. }
\end{aligned}
$$

For a horizontally orientated surface, the curvature is written as,

$$
\kappa=\nabla \cdot \boldsymbol{n}
$$

where,

$$
\boldsymbol{n}=\left(-\frac{h_{x}}{\sqrt{1+h_{x}^{2}+h_{y}^{2}}},-\frac{h_{y}}{\sqrt{1+h_{x}^{2}+h_{y}^{2}}}, \frac{1}{\sqrt{1+h_{x}^{2}+h_{y}^{2}}}\right) .
$$

For a fourth-order approximation to the curvature, we must approximate $h_{x}, h_{y}$, $h_{x x}, h_{y y}$ and $h_{x y}$ with fourth-order accuracy. We assume the discretization to each of these terms has the form,

$$
\frac{\partial^{l+m} h\left(x_{i}, y_{j}\right)}{\partial x^{l} \partial y^{m}}=\Delta z \sum_{i^{\prime}=-2}^{2} \sum_{j^{\prime}=-2}^{2} A_{i^{\prime}, j^{\prime}}^{l, m} F_{i^{\prime}, j^{\prime}} .
$$

The coefficients, $A^{l, m}(l=0,1,2$ and $m=0,1,2)$, are determined by the "method of undetermined coefficients" in which one uses the relation $(2.2)$ to relate $F_{i^{\prime}, j^{\prime}}$ to $h$, and the fact that our discretization for the derivatives of $h$ should be exact for the polynomials $h(x, y)=\left(x-x_{i}\right)^{l}\left(y-y_{j}\right)^{m}$ where $l=0 \ldots 4$ and $m=0 \ldots 4$. As a result, one constructs a matrix system of equations with 25 equations and 25 unknowns. For example, in $2 \mathrm{~d}$, one has the following fourth-order approximations,

$$
\begin{gathered}
\frac{\partial h\left(x_{i}\right)}{\partial x} \approx \frac{\Delta y}{\Delta x}\left(\frac{5}{48}\left(F_{-2}-F_{2}\right)+\frac{17}{24}\left(F_{1}-F_{-1}\right)\right) \\
\frac{\partial^{2} h\left(x_{i}\right)}{\partial x^{2}} \approx \frac{\Delta y}{\Delta x^{2}}\left(\frac{-1}{8}\left(F_{-2}+F_{2}\right)+\frac{3}{2}\left(F_{1}+F_{-1}\right)-\frac{11}{4} F_{0}\right) .
\end{gathered}
$$

Remark: For the fourth-order algorithm, a $5 \times 5 \times 13$ stencil is used. A possible concern here is that in underresolved regions, the interface might pass through the stencil more than one time resulting in an erroneous approximation to the curvature. A simple patch (not implemented for any of the results presented in this paper) for this problem would be to locate the interface crossing, in each 13 cell column of data, closest to $z_{k}$ and then delete other interface crossings by looking at where the divided difference,

$$
D f_{i^{\prime}, j^{\prime}, k^{\prime}}=\frac{f_{i^{\prime}, j^{\prime}, k^{\prime}}-f_{i^{\prime}, j^{\prime}, k^{\prime}-1}}{\Delta z}
$$


TABlE 1. Convergence study for computing curvatures from volume fractions of a unit circle in $2 d$. Results for the second-order and fourthorder discretizations are reported.

\begin{tabular}{|l|r|r|r|r|}
\hline$\Delta x$ & max error (2nd) & max error (4th) & avg. error (2nd) & avg. error (4th) \\
\hline $1 / 16$ & 0.0031 & 0.00104 & 0.0019 & 0.00016 \\
\hline $1 / 32$ & 0.0007 & $4.21 \mathrm{E}-5$ & 0.0005 & $7.9 \mathrm{E}-6$ \\
\hline
\end{tabular}

TABLE 2. Convergence study for computing curvatures from volume fractions of a unit sphere in $3 d$. Results for the second-order and fourthorder discretizations are reported.

\begin{tabular}{|l|r|r|r|r|}
\hline$\Delta x$ & max error (2nd) & max error (4th) & avg. error (2nd) & avg. error (4th) \\
\hline $1 / 16$ & 0.050 & 0.03431 & 0.0035 & 0.00081 \\
\hline $1 / 32$ & 0.010 & 0.00060 & 0.0009 & $2.78 \mathrm{E}-5$ \\
\hline
\end{tabular}

changes sign. All volume fractions in the stencil, $f_{i^{\prime}, j^{\prime}, k^{\prime \prime}}$, in which $k^{\prime \prime} \geq k^{\prime} \geq k$ are set to $f_{i^{\prime}, j^{\prime}, k^{\prime}-1}$.

\section{Numerical validation of curvature discretization for a circle}

We check our curvature discretization algorithm for a circle in $2 \mathrm{~d}$ or a sphere in $3 \mathrm{~d}$. In $2 \mathrm{~d}$, we have a unit circle located at the point $(2,2)$ in a $4 \times 4$ domain. In $3 \mathrm{~d}$, we have a unit sphere located at the origin in a $2 \times 2$ domain. Symmetric boundary conditions are used at the borders of the domain. As demonstrated by Tables 1 and 2, we get the appropriate order of accuracy for our high-order height fraction curvature discretization schemes.

\section{Parasitic currents}

In this section we test our implementation of surface tension for the problem of a static $2 \mathrm{~d}$ drop with surface tension. The exact solution for such a problem is that the velocity $\boldsymbol{u}$ is identically zero. In terms of the Ohnesorge number,

$$
O h=\frac{\mu}{\sqrt{\sigma \rho D}}
$$

and assuming constant density and constant viscosity in the drop, the Navier Stokes equations are,

$$
\frac{D \boldsymbol{u}}{D t}=-\nabla p+\frac{1}{O h} \Delta \boldsymbol{u}-\frac{1}{O h} \kappa \nabla H .
$$

We assume the drop is surrounded by a constant pressure void. The numerical simulation uses the second-order coupled levelset and volume-of-fluid (CLSVOF) algorithm described in [20]. We investigate the maximum velocity of our numerical 
TABlE 3. Convergence study for static droplet with surface tension (parasitic currents test). Maximum velocity at $t=250$ is shown. $O h=$ 12000. $\rho_{L}=1$ and $\rho_{G}=0$. Results for the second-order discretization of curvature and the fourth-order discretization are reported.

\begin{tabular}{|l|r|r|}
\hline$\Delta x$ & max. velocity (2nd) & max. velocity (4th) \\
\hline $1.0 / 32$ & $1.4 \mathrm{E}-6$ & $5.5 \mathrm{E}-7$ \\
\hline $1.0 / 64$ & $1.5 \mathrm{E}-7$ & $2.9 \mathrm{E}-8$ \\
\hline
\end{tabular}

method for varying grid resolutions at the dimensionless time $t=250$. The dimensions of our computational grid are $1 \times 1$ with symmetric boundary conditions at all boundaries. A one diameter drop is placed at the origin of our domain. Our tolerance for the pressure solver and viscous solver is $1.0 E-12$. In Table 3 we display results of our grid refinement study for $O h=12000$. We used both the secondorder height fraction algorithm and the fourth-order height fraction algorithm for calculating curvature. Our results indicate at least second-order convergence using both approaches. We remark that the overall results are not expected to be 4th-order accurate when using the 4th-order height fraction curvature discretization since the underlying Navier-Stokes solver is second order. For a reference of previous results for parasitic currents, we refer the reader to work by $[16,7]$. Our results using the fourth-order accurate curvature discretization algorithm crush any doubt about the ability to calculate surface tension using the volume-of-fluid method.

\section{2d axisymmetric test problems}

In this section we validate the second-order height fraction curvature discretization scheme for the problem of (1) surface tension driven drop oscillations, (2) Rayleighcapillary instability, and (3) steady bubble motion. The governing equations are the Navier-Stokes equations for two phase flows,

$$
\begin{gathered}
\rho \frac{D \boldsymbol{U}}{D t}=\nabla \cdot(-p I+2 \mu D)+\rho g \hat{\boldsymbol{z}}-\sigma \kappa \nabla H \\
\nabla \cdot \boldsymbol{U}=0 \\
\frac{D \phi}{D t}=0 \\
\rho=\rho_{L} H(\phi)+\rho_{G}(1-H(\phi)) \\
\mu=\mu_{L} H(\phi)+\mu_{G}(1-H(\phi)) \\
H(\phi)= \begin{cases}1 & \phi \geq 0 \\
0 & \phi<0\end{cases}
\end{gathered}
$$


TABLE 4. Convergence study for zero gravity drop oscillations $\gamma=1 / 2$.

\begin{tabular}{|l|r|r|}
\hline$\Delta r$ & $E_{\text {Amplitude }}^{\text {avg }}$ & $E_{\text {amplitude }}^{\text {max }}$ \\
\hline $3 / 64$ & $\mathrm{~N} / \mathrm{A}$ & $\mathrm{N} / \mathrm{A}$ \\
\hline $3 / 128$ & 0.00073 & 0.00174 \\
\hline $3 / 256$ & 0.00021 & 0.00054 \\
\hline
\end{tabular}

We either use the second-order "single-phase" method described in $[20]\left(\rho_{G}=0\right)$ or the sharp interface "two-phase" method described in $[11,22]\left(\rho_{G}=0.001\right)$.

For the first problem (1), surface tension driven drop oscillations, we compute the evolution of a drop in a void with a surface tension coefficient $\sigma=1 / 2$ and initial perturbation of $\epsilon=0.05$. Table 4 gives the successive errors in amplitude as one refines the computational grid. In Figure 1, we plot the minor amplitude versus time for the three different grid resolutions.

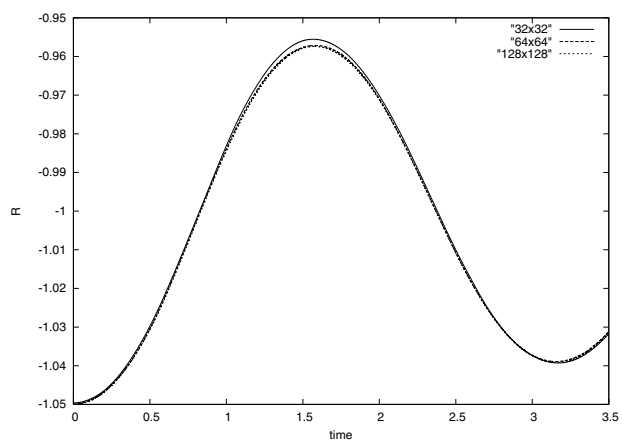

Figure 1. Perturbation in minor amplitude for zero gravity drop oscillations. $\mu_{L}=1 / 50, \gamma=1 / 2$.

For the second problem (2), we calculate the break-up of a liquid jet (in a void) due to capillary instability. The surface tension coefficient is $\sigma=1$ and the viscosity coefficient is $\mu=1 / 200$. In Figure 2, we display the results of our computations for the capillary jet as it breaks up. In Table 5, we measure the relative errors for the interface and velocity field for grid resolutions ranging from $16 \times 32$ to $64 \times 128$.

For our third test problem (3), we compute the steady state shapes of a gas bubble rising in a viscous Newtonian liquid. For comparison, we use the experimental results found in [2] and [10] and computational results in [19].

As in [2] and [10], we present our computational results in terms of the following dimensionless groups. The Reynolds number $R$, the Eötvös number Eo, and the Morton number $M o$ are defined as follows

$$
R=\frac{\rho L U}{\eta_{L}} \quad E_{o}=\frac{g L^{2} U}{\sigma} \quad M o=\frac{g \eta_{L}^{4}}{\rho \sigma^{3}} .
$$


TABLE 5. Convergence study for the Rayleigh capillary instability problem. Two-phase sharp interface method. $t=80$.

\begin{tabular}{|l|r|r|r|}
\hline grid & $E_{\text {interface }}$ & $E_{\text {Liquid }}^{\text {avg }}$ & $E_{\text {Liquid }}^{\max }$ \\
\hline $16 \times 32$ & $\mathrm{~N} / \mathrm{A}$ & $\mathrm{N} / \mathrm{A}$ & $\mathrm{N} / \mathrm{A}$ \\
\hline $32 \times 64$ & 3.87 & 2.77 & 0.014 \\
\hline 64x128 & 0.62 & 0.84 & 0.003 \\
\hline
\end{tabular}

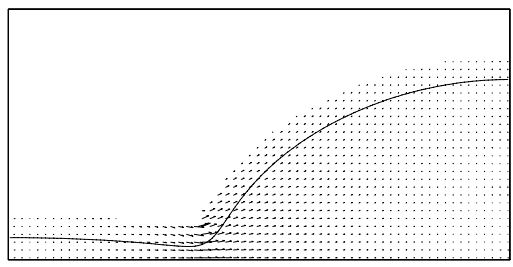

$\mathrm{t}=90.0$

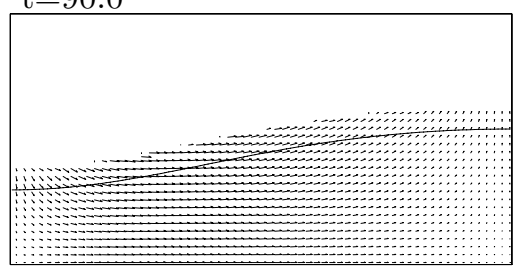

$\mathrm{t}=40.0$
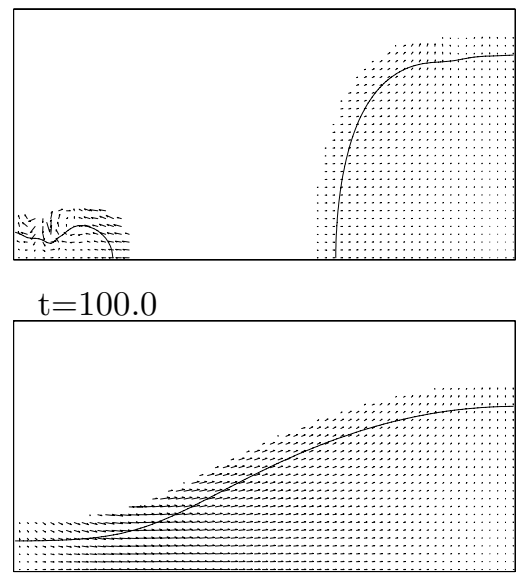

$\mathrm{t}=80.0$

Figure 2. Capillary Instability. Grid resolution is $64 \times 128$.

$\rho$ is the liquid density, $L$ is the bubble diameter, $U$ is a characteristic velocity, $\eta_{L}$ is the liquid viscosity, $\sigma$ is the surface tension, and $g$ is the acceleration of gravity. A comparison of computed terminal bubble shapes versus previous computational and experimental results are reported in Figure 3. Our comparisons include oblate ellipsoidal cap bubbles studied by [2] $\left(E_{o}=243, M_{o}=266\right.$, and $R=7.77$ for bubble figure $2(\mathrm{~d})$ and $E o=116, M o=5.51$, and $R=13.3$ for bubble figure $3(\mathrm{~d}))$, spherical cap bubbles studied by Hnat \& Buckmaster [10] $(R=9.8, M o=0.065$, and $C=4.95$, where $\left.C=\frac{r}{\left(\nu^{2} / g\right)^{1 / 3}}\right)$, and a disk-bubble studied by Ryskin \& Leal [19] $(R=100$ and $W e=10)$.

\section{Conclusions}

The "height fraction" approach for deriving curvature from volume fractions was extended from second-order accurate to fourth-order accurate. The improved accuracy was verified both analytically and through numerical tests. When applied to the "parasitic currents" test, there was a factor of 5 improvement of the fourthorder method over the second-order method. Besides developing a fourth-order 

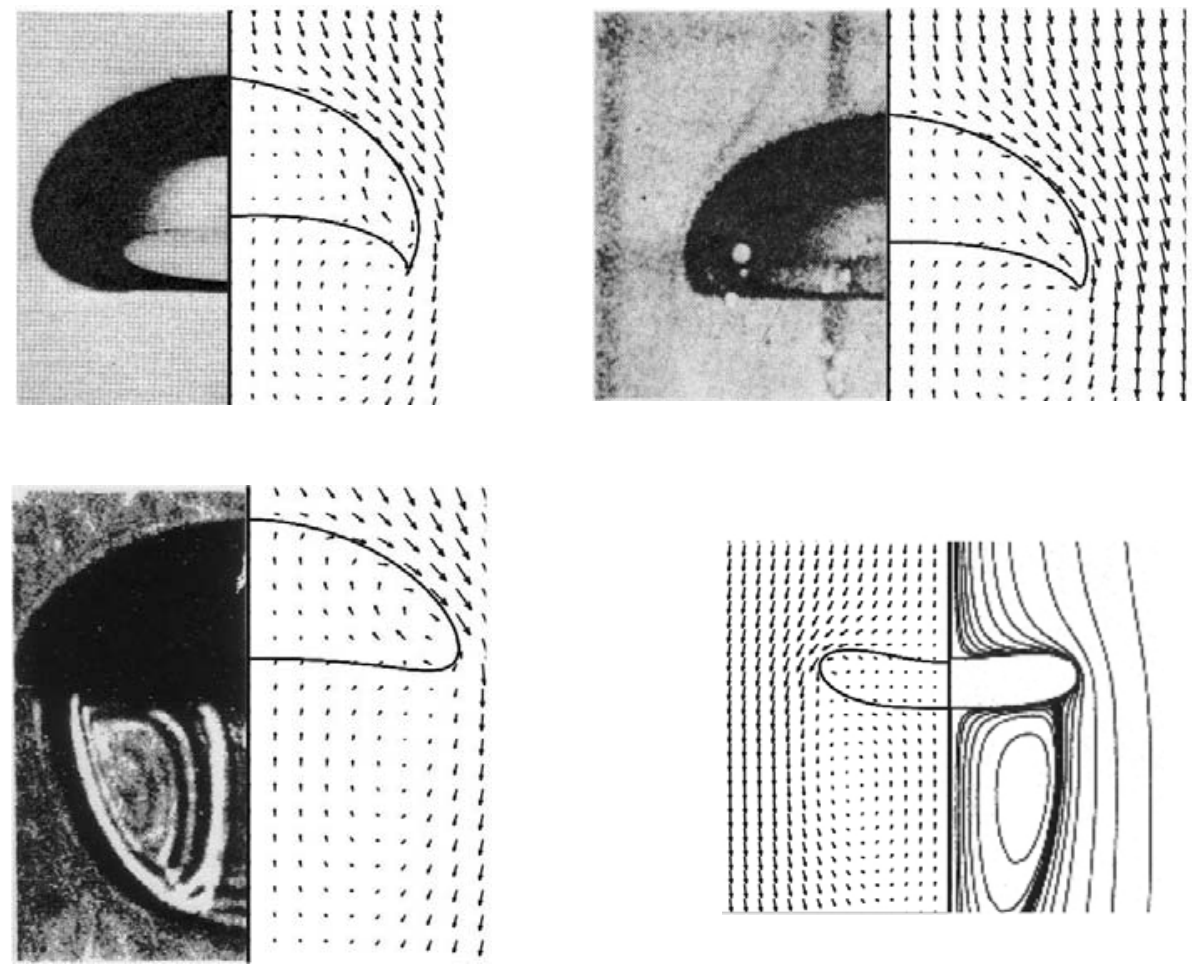

Figure 3. Comparison of numerical results with experimental results and previous computational results. Upper left: Bhaga \& Weber (figure 2, bubble (d)). Upper right: Bhaga \& Weber (figure 3, bubble (d)). Lower left: Hnat \& Buckmaster. Lower right: Ryskin \& Leal.

height fraction technique for finding curvature, we also verified further the robustness of using the second-order height fraction technique together with a 2nd-order Navier-Stokes solver for problems such as the pinch-off of a liquid jet and the steady rise of bubbles.

\section{Acknowledgments}

We thank Mr. D. Kikuchi and Mr. S. Yamaguchi for their help in preparing this manuscript. 


\section{References}

[1] I. Aleinov and E.G. Puckett. Computing surface tension with high-order kernels. In Proceedings of the 6th International Symposium on Computational Fluid Dynamics, Lake Tahoe, CA, 1995.

[2] D. Bhaga and M.E. Weber. Bubbles in viscous liquids: Shapes, wakes and velocities. J. Fluid Mech., 105:61-85, 1981.

[3] J.U. Brackbill, D.B. Kothe, and C. Zemach. A continuum method for modeling surface tension. J. Comput. Phys., 100:335-353, 1992.

[4] A.J. Chorin. Curvature and solidification. J. Comput. Phys., 57:472-490, 1985.

[5] S. Cummins, M. Francois, and D. Kothe. Estimating curvature from volume fractions. Computers and Structures, 83:425-434, 2005.

[6] D. Enright, S. Marschner, and R. Fedkiw. Animation and rendering of complex water surfaces. In SIGGRAPH 2002, volume ACM TOG 21, pages 736-744, 2002.

[7] M. Francois, S. Cummins, E. Dendy, D. Kothe, J. Sicilian, and M. Williams. A balanced-force algorithm for continuous and sharp interfacial surface tension models within a volume tracking framework. J. Comput. Phys., 2005. in press.

[8] D. Gueyffier, J. Li, A. Nadim, R. Scardovelli, and S. Zaleski. Volume of fluid interface tracking with smoothed surface stress methods for three-dimensional flows. $J$. Comput. Phys., 152:423-456, 1999.

[9] J. Helmsen, P. Colella, and E.G. Puckett. Non-convex profile evolution in two dimensions using volume of fluids. LBNL technical report LBNL-40693, Lawrence Berkeley National Laboratory, 1997.

[10] J.G. Hnat and J.D. Buckmaster. Spherical cap bubbles and skirt formation. Physics of Fluids, 19 (2):182-194, 1976.

[11] E. Jimenez, M. Sussman, and M. Ohta. A computational study of bubble motion in newtonian and viscoelastic fluids. Fluid Dynamics and Materials Processing, 2005. (to appear).

[12] M. Kang, R. Fedkiw, and X.-D. Liu. A boundary condition capturing method for multiphase incompressible flow. J. Sci. Comput., 15:323-360, 2000.

[13] H. Liu, S. Krishnan, S. Marella, and H.S. Udaykumar. Sharp interface cartesian grid method ii: A technique for simulating droplet interactions with surfaces of arbitrary shape. J. Computational Physics, 210(1):32-54, 2005.

[14] M.R. Nobari, Y.J. Jan, and G. Tryggvason. Head on collision of drops; a numerical investigation. Technical Report ICOMP-93-45, NASA ICOMP Lewis Research Center, November 1993.

[15] J.Y. Poo and N. Ashgriz. A computational method for determining curvatures. J. Comput. Phys., 84:483-491, 1989.

[16] S. Popinet and S. Zaleski. A front-tracking algorithm for accurate representation of surface tension. International Journal for Numerical Methods in Fluids, 30(6):775793, 1999.

[17] Y. Renardy and M. Renardy. Prost: A parabolic reconstruction of surface tension for the volume-of-fluid method. J. Comput. Phys., 183(2):400-421, 2002.

[18] M. Rudman. A volume tracking method for interfacial flows with large density variations. Int. J. Numer. Methods Fluids, 28:357-378, 1998. 
[19] G. Ryskin and L.G. Leal. Numerical solution of free boundary problems in fluid mechanics. part 2 buoyancy-driven motion of a gas bubble through a quiescent liquid. J. Fluid Mech., 148:19-35, 1984.

[20] M. Sussman. A second-order coupled levelset and volume of fluid method for computing growth and collapse of vapor bubbles. Journal of Computational Physics, 187:110-136, 2003.

[21] M. Sussman and M.Y. Hussaini. A discontinuous spectral element method for the level set equation. J. Scientific Computing, 19:479-500, 2003.

[22] M. Sussman, M.Y. Hussaini, K. Smith, R. Zhi-Wei, and V. Mihalef. A second-order adaptive sharp interface method for incompressible multiphase flow. In Proceedings of the 3rd international conference on Computational Fluid Dynamics, Toronto, Canada, 2004. to appear.

[23] M. Sussman and E.G. Puckett. A coupled level set and volume of fluid method for computing $3 \mathrm{~d}$ and axisymmetric incompressible two-phase flows. J. Comp. Phys., 162:301-337, 2000.

[24] M. Sussman, P. Smereka, and S.J. Osher. A level set approach for computing solutions to incompressible two-phase flow. J. Comput. Phys., 114:146-159, 1994.

[25] H.S. Udaykumar, M.M. Rao, and W. Shyy. Elafint - a mixed Eulerian-Lagrangian method for fluid flows with complex and moving boundaries. Int. J. Numer. Meths. Fluids., 22(8):691-704, 1996.

[26] S.O. Unverdi and G. Tryggvason. A front-tracking method for viscous, incompressible, multi-fluid flows. J. Comput. Phys., 100:25-37, 1992.

[27] M. Williams, D. Kothe, and E.G. Puckett. Convergence and accuracy of kernel-based continuum surface tension models. In Proceedings of the Thirteenth U.S. National Congress of Applied Mechanics, Gainesville, FL, June 16-21 1998.

[28] T. Ye, W. Shyy, and J.N. Chung. A fixed-grid, sharp interface method for bubble dynamics and phase change. J. Comp. Phys., 174:781-815, 2001.

\author{
M. Sussman \\ Department of Mathematics \\ Florida State University \\ Tallahassee, FL 32306, USA \\ e-mail: sussman@math.fsu.edu \\ M. Ohta \\ Department of Applied Chemistry \\ Muroran Institute of Technology \\ 27-1, Mizumotocho, Muroran-shi \\ Hokkaido 050-8585, Japan \\ e-mail: mohta@mmm.muroran-it.ac.jp
}

